\title{
Smart Traffic Light System with Over-speed Detection
}

\author{
$1^{\text {st }}$ Ashley Vijayakumar \\ VLSI and Embedded System ER\&DCI Institute of \\ Technology Thiruvananthapuram, India
}

\author{
$2^{\text {nd }}$ Kadar A A \\ VLSI and Embedded System ER\&DCI Institute of \\ Technology Thiruvananthapuram, India
}

\author{
$3^{\text {rd }}$ Divya D S \\ VLSI and Embedded System ER\&DCI Institute of Technology \\ Thiruvananthapuram, India
}

\begin{abstract}
Congestion in traffic is a serious problem we face nowadays. Although it seems to pervade everywhere, mega cities are those most suffering from it. It is hence necessary to under- stand the road traffic density in realtime for better signal control and effective traffic management. There are often different causes of congestion in traffic like insufficient capacity, unrestrained demand, large Red Light delays etc. While insufficient capacity and unrestrained demand are somewhere interrelated, the delay of respective light is tough-coded and not hooked into traffic. Therefore the necessity for simulating and optimizing control to accommodate this increasing demand arises. However, the synchronization of multiple traffic signal systems at adjacent intersections may be a complicated problem given the varied parameters involved. Conventional systems don't handle variable flows approaching the junctions. This leads to traffic jam and congestion. In this project, a system based on Arduino that evaluates the traffic density using IR sensors and accomplishes dynamic timing slots with different levels is proposed. Also, a reckless driving alert system is to be implemented whereby the nearby traffic police are given an alert message when an over- speeding vehicle is detected.
\end{abstract}

Index Terms-Traffic Light System, Traffic density, Overspeed detection, GSM module

\section{INTRODUCTION}

Traffic Lights or Traffic Signals are signaling devices that are used to control the flow of traffic. Generally, they are positioned at junctions, intersections, ' $\mathrm{X}$ ' roads, pedestrian crossings etc. and alternate the priority of who has to wait and who has to go. The traffic lights will provide instructions to the users (drivers and pedestrians) by displaying lights of standard color. The three colors used in traffic lights are Red, Yellow and Green.

The system must be used to control the traffic lights for smooth and safe movement of traffic. These control systems consists of electro mechanical controllers with clockwork mechanisms or modern solid state computerized systems with easy setup and maintenance. The main problem faced by people nowadays is traffic block, which can be controlled to a great extent by making our traffic light control a smart one. This project aims at controlling the traffic light based on vehicle density rather than providing a fixed time to each. Also, in the existing system, over-speeding vehicles are identified and penalized. But if immediate actions are taken, it will help to control accidents. Hence this project brings about the idea whereby in case of an overspeeding vehicle, the nearby police on duty will be given an alert message. This is implemented with the help of IR modules and GSM.

\section{LITERATURE SURVEY}

The current traffic control systems (TCS) within the metro cities of India are inefficient due to randomness within the traffic density pattern throughout the day. The traffic light timers have a hard and fast period to modify traffic between different directions. Due to this, the vehicles need to wait an extended time albeit the traffic density is extremely less. If the traffic light timer (TST) is often programmed to be manipulated with the continuously varying traffic density, the matter of traffic jam can be often reduced to a significantly lower level.

Bilal Ghazal, et al., in "Smart traffic signal Control System", states that the planning of an intelligent control system is a lively research topic. Researchers round the world are inventing newer approaches and innovative systems to unravel this stressful problem. Models supported by mathematical equations are used to estimate the car waiting time at ajunction, the number of cars and therefore the extension of the waiting cars along the lane, the optimal timing slots for green, yellow, and red lights that best fit the real situation and therefore the efficient combination of routing. The mutual dependencies between nearby intersections cause a sophisticated formulation with cumbersome parameters. These parameters are accidental, hazardous, dependent, and therefore the worse point is that the variance of those parameters with time. Thus, finding a dynamic, consistent, and convenient solution is quite impossible. Researchers from different disciplines are collaborating to explore feasible solutions that reduce traffic jams. Therefore, various methodologies are constantly proposed within the literature and lots of techniques are implemented taking advantage of the technological advances of microcomputers, recent manufactured devices and sensors, innovative algorithms modeling, the maximum amount as possible, the complication of traffic lights. The IR sensors are used in numerous traffic systems. The IR transmitter and therefore the IR receiver are mounted on either side of a road. When an automobile passes on the road between the IR sensors, the system is activated and therefore the car count is incremented. 
The collected information about the traffic density of the various roads of a junction is analyzed to switch dynamically the delays of green light at the lane having the most traffic volume. The entire system might be controlled by PIC microcontroller or maybe by PLC.[1]

Nicole Diaz, et al., in" Smart traffic signal Control System", proposes a system with the subsequent functionalities:

- The traffic signal initially operates like all other traffic signals, with usually fixed times for every state.

- At an intersection of two traffic lights, both are constantly communicating with one another as they're connected to an equivalent network.

- Each traffic signal receives information from the corresponding PIR sensors, during the traditional operation of the traffic signal.

- When the PIR Sensor of a traffic signal captures a vehicle, it transmits a message to the opposite traffic signal, making it change its state to red. The traffic signal that captured the car and sent the message proceeds to vary its state to green.

- Each traffic signal features a minimum duration of green state, that is, if the opposite traffic signal receives a vehicle through its way, but the traffic signal that's in the green state has not yet elapsed its minimum time, the opposite traffic signal will need to wait that minimum time for changing its colour and it'll not do so instantaneously.

- While a traffic signal is in red, the matrix of red LEDs displays the number of remaining seconds before changing to green.

- With the planning of this technique there are a variety of possibilities regarding the operation of the LED Matrix which will be configured consistent with the requirements or preferences desired by the customer, or the intersection during which it'll be placed. [2]

Promila Sinhmar, in "Intelligent traffic signal and Density Control Using IR Sensors and Microcontroller", suggests two approaches, the primary approach - to require data/input/image from object/ subject/vehicle and within the second approach

to process the input file by Computer and Microcontroller and eventually display it on the traffic signal to regulate the closed-loop system system. [3]

\section{A. ARDUINO MEGA 2560}

\section{SYSTEM REQUIREMENTS}

The Arduino Mega 2560, an ATmega2560 based microcontroller. It has 54 digital input/output pins, 16 analog inputs, 4 UARTs, a $16 \mathrm{MHz}$ quartz oscillator, a USB connection, a power jack, a reset button, and an ICSP header. It has got everything needed to support the microcontroller. Simply connect it to a computer using a USB cable or power it with an AC-to-DC adapter or battery to get started. The Mega 2560 board is often programmed with the Arduino Software (IDE).The ATmega2560 has got $256 \mathrm{~KB}$ of flash memory for code storage, $8 \mathrm{~KB}$ of SRAM and $4 \mathrm{~KB}$ of EEPROM. Each of the 54 digital pins on the Mega can be used as an input or output, using the functions: pinMode(), digitalWrite(), and digitalRead(). They operate at 5 volts.

\section{B. LCD DISPLAY}

The LCD is a parallel port and its display regulation is done by controlling several interface pins directly. A register select $(\mathrm{RS})$ pin that controls where within the LCD's memory you're writing data to. A Read/Write $(\mathrm{R} / \mathrm{W})$ pin that selects the mode of operation, an Enable pin that permits writing to the registers. 8 data pins (D0 -D7). The states of these pins (high or low) are the bits that you're writing to a register once you write, or the values you're reading once you read. There's also a display contrast pin (Vo), power supply pins $(+5 \mathrm{~V}$ and Gnd) and LED Backlight (Bklt+ and Bklt-) pins that you simply can use to power the LCD, control the display contrast, and switch on and off the LED backlight, respectively.

\section{GSM MODULE}

The SIM900A is a complete Dual-band GSM/GPRS solution, which may be embedded within the customer applications. It delivers GSM/GPRS 900/1800MHz performance, as per industrial standards, for voice, SMS, data, and fax during a small form factor and with low power consumption. SIM900A can be used in most space requirements in user applications, especially for slender and compact demand of design.

\section{TRAFFIC LIGHT MODULE}

Traffic light module consists of red, yellow, and green LEDs, each of which has an individual control pin to which the digital high and low signals will be provided, and a common ground. It usually operates at $5 \mathrm{~V}$.

\section{E. IR SENSOR MODULE}

The IR sensor module consists of IR Transmitter and Receiver, an opamp, a variable resistor (Trimmer pot), and an output LED. The IR LED (transmitter) emits light. The IR receiver, a photodiode, conducts when light falls on it. Photo- diode is a semiconductor which has a P-N junction, operated in reverse bias, which means that it starts conducting the current in reverse direction when light falls on it, proportional to the amount of light. This property makes it useful for IR detection.

\section{HARDWARE DESIGN}

Arduino Atmega 2560 is chosen as the controller for the system. It is given a $5 \mathrm{~V}$ power supply. A junction of 4 roads is considered and 1 IR is placed on each to obtain the count. The count is compared on a real time basis which is achieved by the Arduino coding. In order to obtain speed of vehicles, 2 IRs are used. The time taken to cross them and the distance at which they are kept are considered to obtain the speed. GSM is used to alert traffic police nearby in case of a reckless driver. The LCD Display shows the speed of car. The block diagram for the proposed system is as shown in Fig. 1.

The circuit diagram is shown in Fig.2. The control for the traffic light module is given from the Arduino for each individual light. The output of each IR sensor is connected to the Arduino. It is used to obtain the count. Each time an obstacle comes across the IR sensor, the output signal goes low. 


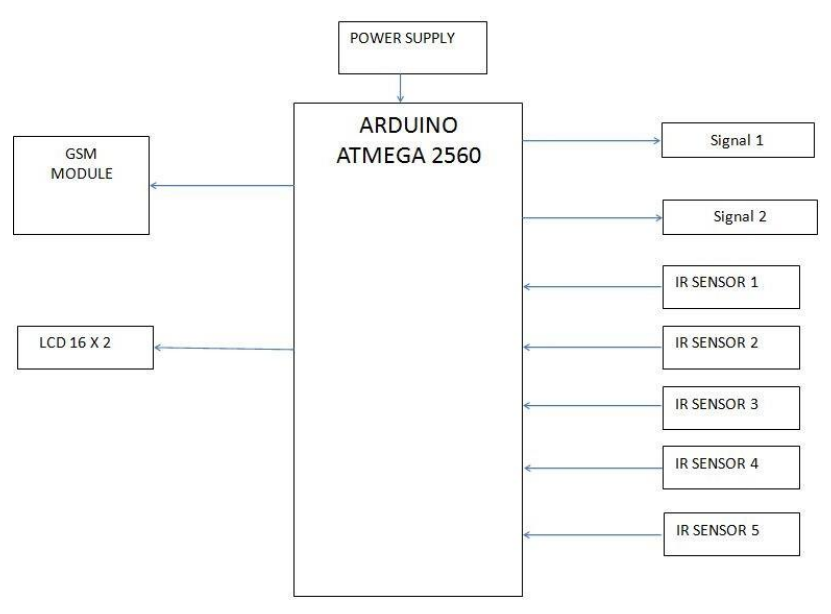

Fig. 1. Block Diagram

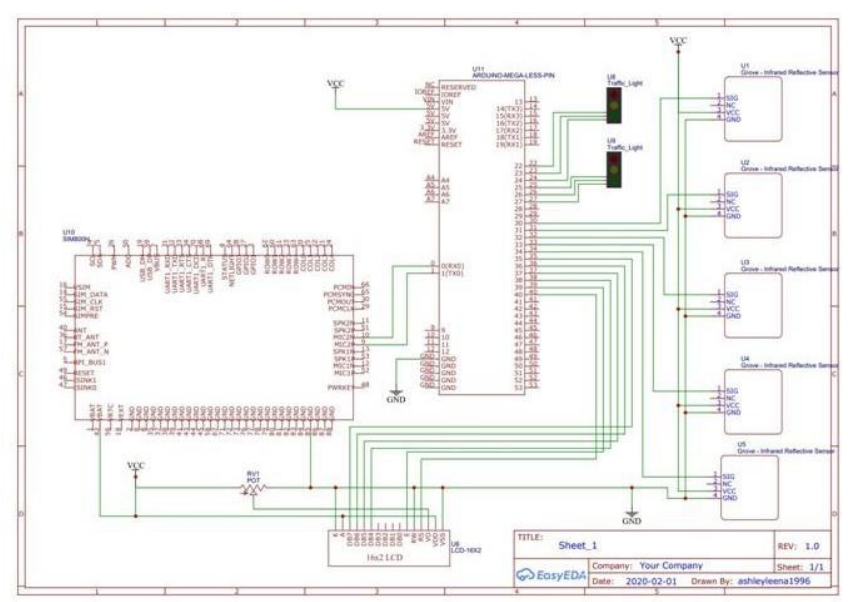

Fig. 2. Circuit Diagram

Transmit and receive signals of GSM are connected to corresponding pins of the controller.

The traffic light configuration is as shown in Table I. The duration of yellow light will be programmed to be for a much shorter period than that for yellow. The vehicle density per lane will be considered by taking the number of vehicles in that lane when it's corresponding light turns red.

TABLE I

TRAFFIC LIGHT CONTROL

\begin{tabular}{|c|l|l|l|l|l|}
\hline \multicolumn{3}{|c|}{ Traffic Light 1 } & \multicolumn{3}{c|}{ Traffic Light 2 } \\
\hline Red & Yellow & Green & Red & Yellow & Green \\
\hline Off & Off & On & On & Off & Off \\
\hline Off & On & Off & On & Off & Off \\
\hline On & Off & Off & Off & Off & On \\
\hline On & Off & Off & Off & On & Off \\
\hline
\end{tabular}

\section{RESULT AND CONCLUSION}

In this project we aim at controlling traffic based on vehicle density and to alert traffic police on reckless driving. The count of each road was obtained when the corresponding light turns red. A comparison of the values is done to figure out the denser road, based on which extra time will be allotted. After each comparison, the values will be reset to obtain the real time comparison.

In the second part, the velocity of vehicles passing through the road is calculated. The speed limit is set at $70 \mathrm{~km} / \mathrm{h}$. For every vehicle passing by, the speed will be displayed in an LCD. In case it exceeds the limit, a buzzer sounds to alert the driver. A message is sent to the nearby police on duty indicating the location from which they are heading and the speed. In case the driver has not slowed down, this will help the police to stay alert and catch the driver.

1) TRAFFIC CONTROL: The traffic light control achieved

is given in Fig. 3. It is implemented using a traffic light module.

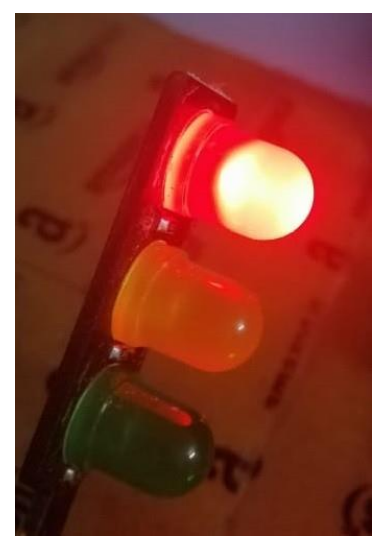

Fig. 3. Traffic Light

2) VEHICLE COUNT: The count obtained per road is obtained as shown in Fig. 4. When the light turns red, the count of corresponding roads are taken and the counts are compared.

Count $4: 10$
C2: 5
Count $4: 11$
C2: 6
Count $2: 3$
C2: 7
Count $2: 4$
C2: 8
Count $4: 12$
C2: 9
Count $2: 5$
C2: 1
Count $3: 6$
C1: 1
Count $3: 7$
C1: 2
Count $3: 8$
C1: 3
Fig. 4 . Count per Road


3) OVERSPEED DETECTION: In Fig. 5, speed obtained and whether message is sent for over speed can be observed.

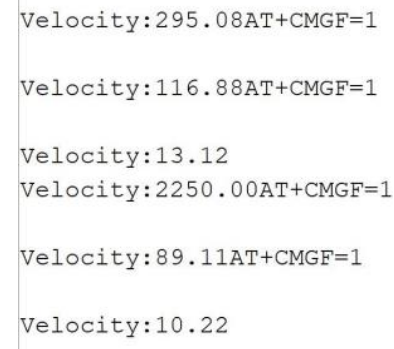

Fig. 5. Over speed Detection

4) SPEED DISPLAY: Display of speed is given in Fig. 6.

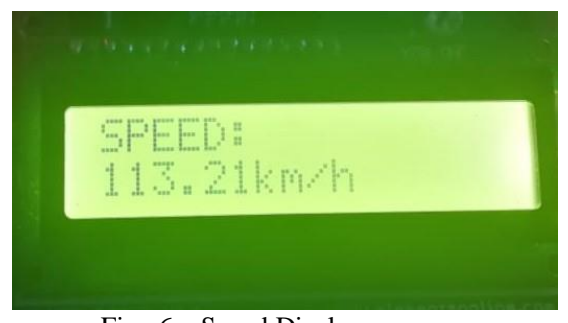

Fig. 6. Speed Display

5) ALERT MESSAGE: The alert message sent gives the location and the speed recorded as shown in Fig. 7.

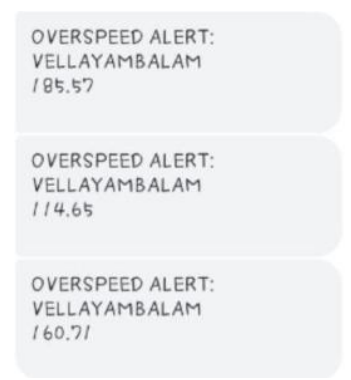

Fig. 7. Alert Message

\section{FUTURE SCOPE}

In future, the brightness of traffic light can be controlled based on the brightness of surrounding light, to make it more energy efficient.

Also, for the elderly and disabled ones, more crossing time may be provided. This can be implemented using RFID whereby those individuals will be given the card. When these cards are tapped on readers provided, crossing time may be extended.

Automatic street lighting can be made associated with this to make it more energy efficient too. When the presence of a vehicle or person id detected, the lights can be made brighter.

For over-speeding, image processing may be used in future projects to make it more accurate.

\section{REFERENCES}

[1] Bilal Ghazal, Khaled EIKhatib, Khaled Chahine, Mohamad Kher- fan,"Smart Traffic Light Control System", Third International Conference on Electrical, Electronics, Computer Engineering and their Applications (EECEA), IEEE 2016

[2] Nicole Diaz, Jorge Guerra, Juan Nicola,"'Smart Traffic Light Control System", IEEE Third Ecuador Technical Chapters Meeting (ETCM), 2018

[3] P. Sinhmar,'Intelligent traffic light and density control using IR sensors and microcontroller", International Journal of Advanced Technology \& Engineering Research (IJATER),Vol. 2, Issue 2, pp. 30-35, March 2012 